25 (1967).

${ }^{6}$ Manufactured by Oak Ridge Technical Enterprises Corporation, Oak Ridge, Tenn.

${ }^{7} \mathrm{~J}$. T. Routti and S. G. Prussin, University of California Lawrence Radiation Laboratory Report No. UCRL-17672, 1968 (unpublished)。

${ }^{8}$ T. Sikkeland and D. F. Lebeck, unpublished work.

${ }^{9}$ V. L. Mikheev, V. I. Ilyushchenko, M. B. Miller, S. M. Polikanov, G. N. Flerov, and Yu. P. Kharitonov, At. Energ. (U.S.S.R.) 22, 90 (1967) [translation: Soviet J. At. Energy 22, 93 (1967)].

${ }^{10}$ G. N. Flerov, S. M. Polikanov, V. L. Mikheev, V. I.
Ilyushchenko, V. F. Kushniruk, M. B. Miller, A. M. Sukhov, and V. A. Shchegolev, Yadern Fiz. $\underline{5}, 1186$ (1967) [translation: Soviet J. Nucl. Phys. $\underline{5}, 848$ (1967)].

${ }^{11}$ M. Nurmia, K. Eskola, P. Eskola, and A. Ghiorso, University of California Lawrence Radiation Laboratory Report No. UCRL-18712, 1969 (unpublished).

${ }^{12}$ P. B. Price and R. M. Walker, Phys. Rev. Letters $\underline{3}, 113$ (1962).

${ }^{13}$ S. G. Nilsson, University of California Lawrence Radiation Laboratory Report No. UCRL-18355, Revised 1968 (unpublished).

\title{
EVIDENCE FOR DISCOVERY OF GRAVITATIONAL RADIATION*
}

\author{
J. Weber \\ Department of Physics and Astronomy, University of Maryland, College Park, Maryland 20742
}

(Received 29 April 1969)

\begin{abstract}
Coincidences have been observed on gravitational-radiation detectors over a base line of about $1000 \mathrm{~km}$ at Argonne National Laboratory and at the University of Maryland. The probability that all of these coincidences were accidental is incredibly small. Experiments imply that electromagnetic and seismic effects can be ruled out with a high level of confidence. These data are consistent with the conclusion that the detectors are being excited by gravitational radiation.
\end{abstract}

Some years ago an antenna for gravitational radiation was proposed. ${ }^{1}$ This consists of an elastic body which may become deformed by the dynamic derivatives of the gravitational potentials, and its normal modes excited. Such an antenna measures, precisely, the Fourier transform of certain components of the Riemann curvature tensor, averaged over its volume. The theory has been developed rigorously, starting with Einstein's field equations to deduce $e^{2}$ equations of motion. Neither the linear approximation nor the energy-flux relations are needed to describe these experiments, but their use enables discussion in terms of more familiar quantities. All aspects of the antenna response and signal-tonoise ratio can be written in terms of the curvature tensor. The theory was verified experimentally by developing a high-frequency source ${ }^{3}$ and producing and detecting dynamic gravitational fields in the laboratory.

Several programs of research are being carried out. One employs laboratory masses in the frequency range $1-2 \mathrm{kHz}^{4}$ Another is concerned with expected gravitational radiation from the pulsars. ${ }^{5}$ Some designs for such antennas suggest a pulsar detection range approaching 1000 pc. A third class of antennas employs the quadrupole modes of the earth, ${ }^{1}$ the moon, and planets $^{6}$ for the range $1 \mathrm{cycle} / \mathrm{h}$ to $1 \mathrm{cycle} / \mathrm{min}$. This array is a new set of windows for studying the universe.

Search for gravitational radiation in the vicinity of $1660 \mathrm{~Hz}$. A frequency in the vicinity of 1660 $\mathrm{Hz}$ was selected because the dimensions are convenient for a modest effort and because this frequency is swept through during emission in a supernova collapse. It was expected that once the technology was refined, detectors could be designed for search for radiation from sources with radio or optical emission, such as the pulsars. A knowledge of the expected frequency and $Q$ of a source enormously increases the probability of successful search.

However, occasional signals were seen at 1660 $\mathrm{Hz}$ and small numbers of coincidences were observed on detector $\mathrm{s}^{7,}{ }^{8}$ separated by a few kilometers. To explore these phenomena further, larger detectors were developed. One of these is now operating at Argonne National Laboratory. My definition of a coincidence is that the rectified outputs of two or more detectors cross a given threshold in the positive direction within a specified time interval. For the present experiments the time interval was $0.44 \mathrm{sec}$. The magnitudes of the outputs at a coincident crossing enable computation of the probability that the coincidence was accidental. Observation of a number of coincidences with low probability of occurring 


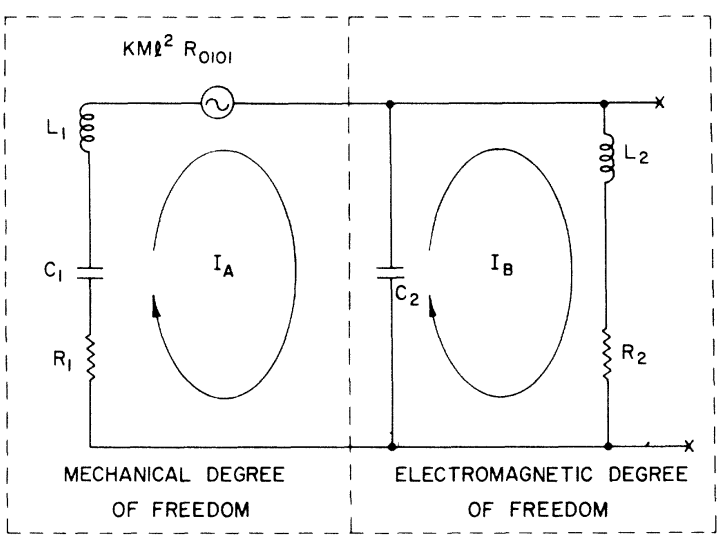

FIG. 1. Equivalent circuit of gravitational-radiation detector with piezoelectric coupling and external inductance.

statistically establishes, with good confidence, that the detectors are being excited by a common source. We may conclude that such coincidences are due to gravitational radiation if we are certain that other effects such as seismic and electromagnetic disturbances are not exciting the detectors.

Detectors. - Six detectors have been operating. Four of them are aluminum cylinders of $153-\mathrm{cm}$ length tuned to a narrow band of frequencies ( $\Delta \omega$ $\approx 0.1 \mathrm{rad} \mathrm{sec}^{-1}$ ) near $1660 \mathrm{~Hz}$. The bandwidth is adjustable. Piezoelectric crystals bonded to their surfaces couple the normal-mode oscillations to an electromagnetic degree of freedom. Figure 1 is an equivalent circuit. The Riemann tensor is the driving voltage. One cylinder has a diameter of $96 \mathrm{~cm}$ and employs cryogenically cooled electronics. There are two cylinders with $66-\mathrm{cm}$ diameters and one with $61-\mathrm{cm}$ diameter. These have completely new (and as yet, unpublished) room-temperature instrumentation. One of the $66-\mathrm{cm}$ detectors is at the Argonne National Laboratory; all other detectors are at the University of Maryland.

A telephone line transmits the radiofrequency output of the Argonne detector to Maryland. This output and that of the Maryland 66- $\mathrm{cm}$ detector are coupled to a two-channel coincidence detector. Pen and ink recorders with event markers record coincidences on all charts.

The high- $Q$ mechanical systems begin oscillating as soon as they are excited. The decay is determined by the relaxation rate. The time required for the electromagnetic output to build up is governed by the electromagnetic degree of freedom (Fig. 1) and associated electronic instrumentation. It is an extremely exacting task to ob- tain good noise performance with short time resolution. A time resolution smaller than $0.30 \mathrm{sec}$ was achieved with the $66-\mathrm{cm}$ detectors at a point in the instrumentation where somewhat less than half of the noise power was originating in the mechanical degree of freedom, and this output was coupled to the coincidence detector. Further processing provided an output with essentially all fluctuations due to the cylinder Brownian motion. The thermal-fluctuation-noise limit implies a relative displacement of the end faces roughly $10^{-14} \mathrm{~cm}$, strains of parts in $10^{16}$.

The coincidence thresholds can be crossed in more than one way. If the detector output suddenly increases, threshold will be crossed. However, if the detector output is somewhat high, threshold crossing due to other sources of noise becomes highly probable. Calculations show that the probability of a coincidence occurring accidently is about the same for both cases. The use of fast recorder speeds established that most of the crossings did in fact occur at the leading edge. An earlier ${ }^{7,8}$ experiment showed both types of coincidence.

The telephone line linking the two detectors is very convenient but not absolutely necessary. Recorders at both locations could, by later comparison, establish that coincidences are being observed. The telephone line from Argonne to Chicago is by wire and repeater, then via microwave relay links to Providence, Rhode Island, then via wire and repeaters to Maryland. Measurements established that the two-way travel time for electromagnetic signals was roughly 25 msec. An initial experiment utilized a two-way telephone to excite the mechanical mode of both detectors from Maryland to prove that the complete system records coincidences. Thereafter telephone communications were one way.

Results. - Table I lists some of the coincidences together with the period of time which must elapse for a coincidence with equal to or greater amplitudes to occur accidentally. This time for accidental coincidence is obtained in the following way. For each coincidence the amplitudes are recorded. A study of the chart records for the given day shows that the coincidence amplitude for a given detector $A$ is exceeded $N_{A}$ times and for detector $B, N_{B}$ times that day. Let $\tau$ be the coincidence resolving time and $T$ the length of the day. The probability $P_{A B}$ that the coincidence was accidental is

$$
P_{A B}=4 N_{A} N_{B} \tau^{2} / T^{2}
$$


Table I. Argonne National Laboratory and University of Maryland detector coincidences. Time resolution for the two $66-\mathrm{cm}$ detectors is $0.4 \mathrm{sec}$. The $61-$ and $96-\mathrm{cm}$ detectors are not coupled to a coincidence counter. Their threshold crossing time is not accurately known and this is taken into account in computing the frequency of accidental three- and four-detector coincidences.

\begin{tabular}{|c|c|c|c|c|}
\hline \multirow{3}{*}{$\begin{array}{c}\text { DATE } \\
\text { MONTH/DAY/YEAR } \\
12 / 30 / 68^{\circ}\end{array}$} & \multirow{3}{*}{$\begin{array}{c}\begin{array}{c}\text { UNIVERSAL } \\
\text { TIME }\end{array} \\
1033\end{array}$} & \multicolumn{2}{|c|}{$\begin{array}{l}\text { NUMBER OF TIMES PER DAY } \\
\text { COINCIDENCE AMPLITUDE IS EXCEEDED }\end{array}$} & \multirow{3}{*}{$\begin{array}{c}\text { PERIOD PER } \\
\text { ACCIDENTAL COINCIDENCE } \\
\text { 18 YEARS }\end{array}$} \\
\hline & & MARYLAND 66 CM DETECTOR & ARGONNE 66 CM DETECTOR & \\
\hline & & 25 & 15 & \\
\hline $1 / 1 / 69^{b}$ & 0052 & 6 & 88 & $8 \times 10^{4}$ YEARS \\
\hline $1 / 6 / 69^{b}$ & 0025 & 110 & 4 & 230 YEARS \\
\hline $1 / 28 / 69$ & 1546 & 24 & 5 & 720 DAYS \\
\hline $1 / 30 / 69$ & 1656 & 1 & 5 & 48 YEARS \\
\hline $2 / 5 / 69^{\circ}$ & 2221 & 30 & 30 & 7 YEARS \\
\hline $2 / 6 / 69$ & 0447 & 150 & 4 & 144 DAYS \\
\hline $2 / 16 / 69$ & 0130 & $20\}$ & $72\}$ & $3 \times 10^{4}$ YEARS \\
\hline $2 / 16 / 69$ & $0130.5 \mathrm{~S}$ & $200^{3}$ & $200^{3}$ & \\
\hline $2 / 16 / 69$ & 0159 & 1 & 24 & 10 YEARS \\
\hline $2 / 21 / 69$ & 0634 & 26 & 12 & 280 DAYS \\
\hline $2 / 23 / 69^{\circ}$ & 1218 & 40 & 12 & 15 YEARS \\
\hline $3 / 4 / 69$ & .0913 & 30 & 15 & 190 DAYS \\
\hline $3 / 15 / 69$ & 0341 & 75 & 6 & 190 DAYS \\
\hline $3 / 20 / 69^{\circ}$ & $\left.1741 \frac{1}{2}\right\}$ & $140\}$ & 96 & $7 \times 10^{7}$ YFARS \\
\hline $3 / 20 / 69^{\circ}$ & $1744\}$ & $\infty^{\}}$ & $125\}$ & $7 \times 10$ TEAKS \\
\hline $3 / 21 / 69^{b}$ & 0311 & 48 & 2 & $4 \times 10^{4}$ YEARS \\
\hline
\end{tabular}

aTriple coincidence.

The number of days required for the coincidence to occur accidentally is

$$
n=T / 2 N_{A} N_{B} \tau \text {. }
$$

During an 81-day period there were more than 17 significant two-detector coincidences, five three-detector coincidences, and three four-detector coincidences. It is important that the twodetector coincidences alone establish the high probability that the coincidences reported here are not accidental. The smallest signal two-detector coincidence which is listed could have occurred accidentally once in 144 days, while the largest signal two-detector coincidence could have occurred accidentally once in 48 years. The three- and four-detector coincidences have far smaller probability of accidental occurrence, based on calculations in accordance with the caption on Table I. The double two-detector coincidence on 16 February 1969 has a probability of accidental occurrence every $3 \times 10^{4} \mathrm{yr}$. The pair of triple-detector coincidences on 20 March 1969 has a probability of occurring accidentally every $7 \times 10^{7} \mathrm{yr}$. Therefore it is quite certain that all of the coincidences cannot be accidental.
${ }^{\mathrm{b}} \mathrm{Quadruple}$ coincidence.

None of the coincident signals was far above the thermal fluctuations. With signal-to-noise ratio roughly unity, external excitation can result in any power output between zero and several times the noise power at coincidence, depending upon the amplitude and phase of the noise at the moment of coincidence. Chart records for an equal-amplitude coincidence are shown in Fig. 2. For most of the observing time only the two 66$\mathrm{cm}$ detectors were fully operational. A study of the records shows that on some occasions the Argonne output was high while the other $66-\mathrm{cm}$ detector was not far from the mean, and one or both of the remaining $1660-\mathrm{Hz}$ detectors also had well above-mean output. This is expected for excitations comparable with the thermal fluctuations. It is also expected and observed that some detectors will have statistically low output at a two-detector coincidence.

A plot of all significant events versus sidereal time shows activity in all quadrants. The "antenna" beamwidth is about $70 \mathrm{deg}$ in azimuth centered on the meridian, and it is isotropic in the plane of the meridian. This leaves many possibilities open for the source or sources. 

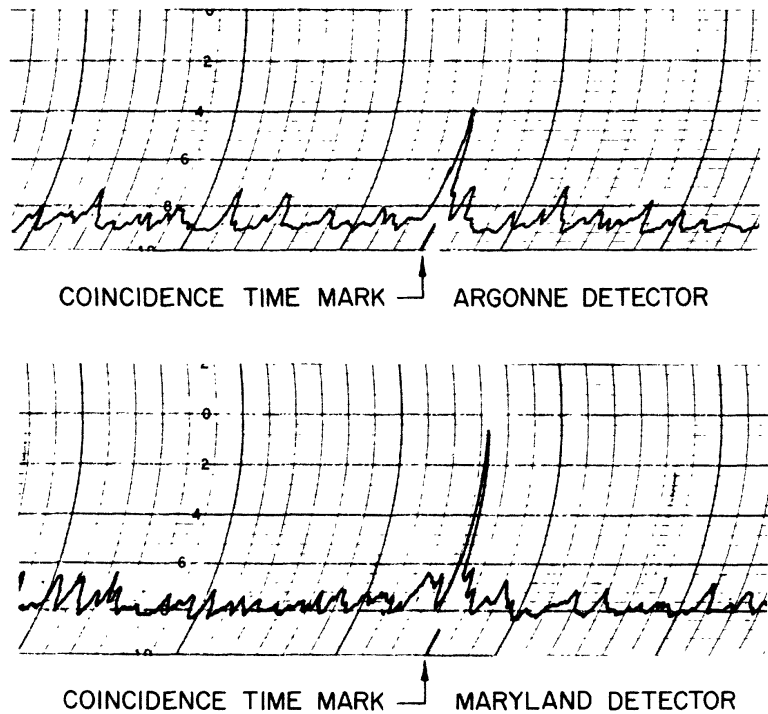

FIG. 2. Argonne National Laboratory and University of Maryland detector coincidence.

Detector response.-Consider the $96-\mathrm{cm}$ detector (Fig. 1) with cryogenically cooled electronics. The electromagnetic and electromechanical degrees of freedom both have long relaxation times. For simplicity assume for the moment that the detector is lossless. Then one normal mode of frequency $\omega_{1}$ can be shown to require

$$
I_{B}=\left(L_{1} / L_{2}\right)^{1 / 2} I_{A},
$$

and the other normal mode, of frequency $\omega_{2}$, requires

$$
I_{B}=-\left(L_{1} / L_{2}\right)^{1 / 2} I_{A}
$$

Suppose the Riemann tensor is a delta function at $t=0$, then (3) and (4) require for $t<0$,

$$
\begin{aligned}
& I_{A}=0, \\
& I_{B}=0 ;
\end{aligned}
$$

and for $t>0$, with $R$ an appropriate constant,

$$
\begin{aligned}
I_{A}= & R\left[\cos \omega_{1} t+\cos \omega_{2} t\right], \\
I_{B}= & 2 R\left[L_{1} / L_{2}\right]^{1 / 2} \\
& \times\left\{\sin \left[\frac{1}{2}\left(\omega_{1}+\omega_{2}\right) t\right] \sin \left[\frac{1}{2}\left(\omega_{2}-\omega_{1}\right) t\right]\right\}
\end{aligned}
$$

Equation (6B) implies that the electromagnetic output will start from zero and not reach a max- imum until a time

$$
\Delta t \approx\left(\omega_{2}-\omega_{1}\right)^{-1} \pi
$$

$\Delta t$ is $11 \mathrm{sec}$ for the $96-\mathrm{cm}$ detector. Taking loss into account does not significantly affect this result for the very small electromagnetic loss associated with a cryogenically cooled instrument. If the initial values of the currents are zero, the 96- $\mathrm{cm}$ detector would be expected to develop maximum electromagnetic output $11 \mathrm{sec}$ after impulsive excitation of its mechanical system. This delay will of course be absent if the electromagnetic degree of freedom is impulsively excited.

The other detectors do not employ cryogenically cooled electronics and their electromagnetic degrees of freedom have relaxation times $<10^{-4}$ sec. Their output builds up in less than $0.1 \mathrm{sec}$ after excitation of their mechanical systems.

If no noise were present we would expect then to see coincidences on the 66- and $61-\mathrm{cm}$ detectors, followed $11 \mathrm{sec}$ later by a $96-\mathrm{cm}$ detector peak. The delayed response has been observed for about $\frac{1}{4}$ of the events. This is good evidence that the excitation was not some spurious electromagnetic signal which found its way into the system through the shields and cables. The presence of noise modifies this argument in several ways. We cannot always expect to see this delay. In addition, the noise of the electromagnetic degrees of freedom might trigger coincidences for any pair of detectors which are close to threshold as a result of somewhat earlier mechanical system excitation. In the earlier experiment ${ }^{7,8}$ over a 2-km baseline some coincidences were observed accompanied by the expected delay and others appeared simultaneous as a result of electromagnetic degree of freedom triggering the already excited mechanical systems.

Mr. Reginald Clemens and I have analyzed in detail the response of a detector having one degree of freedom to gravitational radiation originating in a supernova collapse. We found that the highest $Q$ detectors have best response with oscillations building up within milliseconds following arrival of the gravitational signal. Early in 1968, Mr. Clemens extended this analysis to the case of two degrees of freedom and found the output-response-time delay for long-relaxationtime electromagnetic systems. The signals reported here appear to be of short duration, but beyond this fact there is no evidence that they are due to collapse of a star system.

Nongravitational effects. - All detectors are mounted on acoustic filters and a seismic array ${ }^{9}$ 
monitors earth motion at the Maryland site. Earthquakes and other earth motions are recorded on the seismic array and are not, in general, seen on the gravitational-radiation detectors. A seismic explanation of the coincidences requires activity in a zone of the earth with travel-time differences of about $\frac{1}{2} \mathrm{sec}$ between Argonne and Maryland. Such a zone has less than $10^{-3}$ of the total Earth volume. The effect of the remainder of the earth would produce a high noise level at each site which is not observed.

The electromagnetic response of each detector was studied and a program of additional shielding undertaken in February, 1969. Shielding sufficient to attenuate electromagnetic signals far below levels then required for coincidence was installed and had no observable effect on coincidence rates.

Only the Argonne and Maryland 66- cm detectors were coupled to the coincidence detector. Extreme care was taken to guarantee that signals from one detector did not cross couple into the other and to insure that switching coincidence marker pens did not excite the detectors. Coincidences were in fact observed between the Maryland 61- and 96- and the Argonne 66-cm detectors which were not coupled to each other in any way. Events were verified by a recorder at Argonne National Laboratory.

Conclusion. - Gravitational-radiation detectors at ends of a $1000-\mathrm{km}$ baseline have occasional coincidences with overwhelming statistics indicating a common origin. The monitoring seismic array and the short time resolution rule out seismic effects. The shielding experiments and the observation of a delayed response of the cryogenically cooled detector rule out electromagnetic excitation, at least for $\frac{1}{4}$ of the coincidences. We must therefore conclude that all of the coincidences are neither accidental nor due to seismic or electromagnetic effects. This is good evidence that gravitational radiation has been discovered.

Excitation at threshold implies a large gravitational-radiation flux-roughly $10^{4}$ ergs $\mathrm{cm}^{-2}$ $\mathrm{sec}^{-1}$, and the signals listed in Table I imply at least a mean gravitational-radiation energy density $\sim 10^{-32} \mathrm{~g} \mathrm{~cm}^{-3}$ over the bandwidth $\Delta \omega \sim 0.1$ $\operatorname{rad~} \sec ^{-1}$.

The present installation is being modified to achieve a time resolution $3 \times 10^{-2} \mathrm{sec}$. Search at wider bandwidths and other frequencies can be achieved with relatively minor modifications. There are also available cylinder modes which would be excited by the scalar ${ }^{2,10,11}$ components of the fields for possible tests of the BransDicke-Jordan scalar-tensor theories of gravitation.

The great assistance and cooperation of the Argonne National Laboratory was a major factor in carrying out these experiments. Dr. G. Roy Ringo made valuable suggestions. Dr. J. Sinsky supervised manufacture of the aluminum bars and crystals. Mr. Reginald W. Clemens and Mr. Harry Kriemelmeyer assisted with the mechanical designs. Mr. Darrell Gretz constructed most of the electronics with design assistance from Mr. J. Giganti. Mr. J. Peregrin assisted in the detector installation and maintenance. A number of the ideas for these experiments were developed in the stimulating atmosphere of the Aspen Center for Physics, Aspen, Colorado. I thank the Silver Spring, Maryland, and Lemont, Ilinois, engineers of the American Telephone and Telegraph Company Long Lines Department for providing high-quality telephone transmission between Argonne, Illinois, and Maryland. It is my pleasure to acknowledge valuable discussions with C. W. Misner, H. S. Zapolsky, E. F. Beall, R. H. Dicke, F. J. Dyson, G. Westerhout, J. A. Wheeler, and P. G. Bergmann.

\footnotetext{
*Work supported in part by the National Science Foundation.

${ }^{1} \mathrm{~J}$. Weber, Phys. Rev. 117, 306 (1960). See also, J. Weber, General Relativity and Gravitational Waves (Interscience Publishers, Inc., New York, 1961), Chap. 8.

${ }^{2} \mathrm{~J}$. Weber, Relativity Groups and Topology (Gordon and Breach Publishers, Inc., New York, 1964), p. 875.

${ }^{3}$ J. Sinsky and J. Weber, Phys. Rev. Letters 18, 795 (1967); J. Sinsky, Phys. Rev. 167, 1145 (1968).

${ }^{4} \mathrm{~J}$. Weber, Phys. Rev. Letters 17, 1228 (1966).

${ }^{5} \mathrm{~J}$. Weber, Phys. Rev. Letters 21, 395 (1968).

${ }^{6} \mathrm{~J}$. Weber, in Physics of the Moon, edited by S. F. Singer (American Astronautical Society, Hawthorne, Calif., 1967), p. 199.

7J. Weber, Phys. Rev. Letters 18, 498 (1967).

${ }^{8} \mathrm{~J}$. Weber, Phys. Rev. Letters 20,1307 (1968).

${ }^{9}$ J. Weber and J. V. Larson, J. Geophys. Res. 71, 6005 (1966).

${ }^{10}$ R. H. Dicke, Ref. 2, p. 165.

${ }^{11} \mathrm{D}$. C. Robinson and J. Winicour, Phys. Rev. Letters 22, 198 (1969).
} 